\title{
BMJ Global Health Using health management information system data: case study and verification of institutional deliveries in Ethiopia
}

\author{
Catherine Arsenault (D) , ${ }^{1}$ Bereket Yakob, ${ }^{2}$ Munir Kassa, ${ }^{3}$ Girmaye Dinsa, ${ }^{2,4}$ \\ Stéphane Verguet (iD ${ }^{1}$
}

\begin{abstract}
To cite: Arsenault C, Yakob B, Kassa M, et al. Using health management information system data: case study and verification of institutional deliveries in Ethiopia. BMJ Global Health 2021;6:e006216. doi:10.1136/ bmjgh-2021-006216
\end{abstract}

Handling editor Seye Abimbola

Received 5 May 2021 Accepted 3 August 2021
A) Check for updates

(c) Author(s) (or their employer(s)) 2021. Re-use permitted under CC BY. Published by BMJ.

${ }^{1}$ Department of Global Health and Population, Harvard T.H. Chan School of Public Health, Boston, Massachusetts, USA ${ }^{2}$ Fenot project, School of Population and Public Health, University of British Columbia, Addis Ababa, Ethiopia

${ }^{3}$ Minister's Office, Ministry of Health, Addis Ababa, Ethiopia ${ }^{4}$ Department of Public Health and Health Policy, Haramaya University, Hararamaya, Ethiopia

Correspondence to Dr Catherine Arsenault; carsenault@hsph.harvard.edu

\section{ABSTRACT}

Health management information systems (HMIS) are a crucial source of timely health statistics and have the potential to improve reporting in low-income countries. However, concerns about data quality have hampered their widespread adoption in research and policy decisions. This article presents results from a data verification study undertaken to gain insights into the quality of HMIS data in Ethiopia. We also provide recommendations for working with HMIS data for research and policy translation. We linked the HMIS to the 2016 Emergency Obstetric and Newborn Care Assessment, a national census of all health facilities that provided maternal and newborn health services in Ethiopia. We compared the number of visits for deliveries and caesarean sections (Csections) reported in the HMIS in 2015 (January-December) to those found in source documents (paper-based labour and delivery and operating theatre registers) in 2425 facilities across Ethiopia. We found that two-thirds of facilities had 'good' HMIS reporting for deliveries (defined as reporting within $10 \%$ of source documents) and half had 'very good' reporting (within $5 \%$ of source documents). Results were similar for reporting on $\mathrm{C}$-section deliveries. We found that good reporting was more common in urban areas (OR: 1.30, $95 \% \mathrm{Cl} 1.06$ to 1.59 ), public facilities (OR: $2.95,95 \% \mathrm{Cl} 1.38$ to 6.29) and in hospitals compared with health centres (OR: $1.71,95 \% \mathrm{Cl} 1.13$ to 2.61). Facilities in the Somali and Afar regions had the lowest odds of good reporting compared with Addis Ababa and were more likely to over-report deliveries in the HMIS. Further work remains to address remaining discrepancies in the Ethiopian HMIS. Nonetheless, our findings corroborate previous data verification exercises in Ethiopia and support greater use and uptake of HMIS data for research and policy decisions (particularly, greater use of HMIS data elements (eg, absolute number of services provided each month) rather than coverage indicators). Increased use of these data, combined with feedback mechanisms, is necessary to maintain data quality.

\section{INTRODUCTION}

Health management information systems (HMIS), based on data reported by health facilities, are an important source of health statistics in low-income and middle-income countries (LMICs). Well-functioning HMIS can provide near real-time data on the

\section{Summary box}

Using data from source documents (paper-based registries) in 2425 facilities in Ethiopia, we found that $66 \%$ of facilities had good health management information systems (HMIS) reporting for deliveries (defined as reporting within $10 \%$ of source documents), and half had very good reporting (within $5 \%$ of source documents).

- Hospitals, public facilities and those in urban areas had higher odds of good reporting; facilities in the Somali and Afar regions had the most over-reporting.

- Based on these findings and those of other verification exercises, we argue for greater use of HMIS data elements (eg, absolute number of services provided each month) for research and policy decisions.

- Increased use of these data, combined with feedback mechanisms, is necessary to improve and maintain data quality; country-led HMIS should be the primary source of information for tracking health system performance in low-income and middleincome countries.

performance of health systems and can be used for planning and resource allocation and to track progress on universal health coverage and the Sustainable Development Goals.

The central role of HMIS in improving reporting in LMICs is increasingly recognised. ${ }^{2}$ However, concerns about data quality have hampered the widespread use of HMIS for research and policy decisions across LMICs. In Ethiopia, the Health Sector Transformation Plan (2015-2020) called for a 'data revolution' in the way that health data are collected, analysed and disseminated for decision-making. ${ }^{3}$ Since then, several initiatives have aimed to improve the quality of the Ethiopian HMIS. $^{4-7}$ In 2018, Ethiopia adopted the District Health Information Software 2 (DHIS2), a web-based HMIS platform used in over 70 LMICs, and launched a 
nationwide campaign to improve internet connectivity in health facilities.

There have been few validations of HMIS data in LMICs. ${ }^{5-12}$ Many of these studies have focused on assessing whether intervention coverage indicators estimated using HMIS are consistent with those from household surveys (eg, the demographic and health survey (DHS) ). ${ }^{5911} 13$ Intervention coverage indicators (eg, the proportion of women giving birth in health facilities or the proportion of fully immunised children) require two valid data elements: valid numerators (eg, number of women giving birth in facilities) and valid denominators (eg, total number of women giving birth in a catchment area). In addition to estimating intervention coverage indicators, the HMIS should be used to track individual data elements such as the absolute number of services delivered, the number of consultations taking place, and headcounts, cases and deaths from particular conditions. Monitoring the absolute volume of services provided each month can be used for evaluating the effect of new policies aimed at increasing healthcare use or for identifying potential disruptions in health services caused by health system shocks such as COVID-19. ${ }^{14}$

In Ethiopian health facilities, the number of health services provided on a daily basis is first recorded in paper-based registers (tallies or logbooks) in each department (eg, labour and delivery ward register, operating theatre register, safe abortion register, family planning register, newborn unit register, etc).${ }^{15}$ Data from these paper-based documents are then compiled by a facility statistician (or HMIS focal person) and entered into a digital format for reporting (on a monthly basis for the majority of data). The HMIS focal person is responsible for conducting random accuracy checks and trained in data quality assessments. ${ }^{16}$ It is unclear whether these quality assessments are implemented consistently. ${ }^{15}$

Another way to assess the accuracy of HMIS data is to compare values reported in the HMIS to those found in source documents such as paper-based facility registers. However, studies comparing HMIS with source documents generally require in-person visits, tend to be resource-intensive and thus have generally included small facility samples. For example, similar studies in Ethiopia, Nigeria, Rwanda and Tanzania had samples ranging from only 45 to 400 facilities. ${ }^{68-101718}$ These small samples have prevented meaningful assessments of facility characteristics linked with good HMIS reporting.

Our article presents results from a verification study undertaken to gain insights into the quality of HMIS data in Ethiopia. We also describe our experience working with these data in the context of the Fenot project (Achieving Excellence in Primary Healthcare in Ethiopia). ${ }^{19}$ Working closely with the Ministry of Health and Regional Health Bureaus across Ethiopia, the Fenot project aims to improve the use of locally available evidence for planning and decision-making and to build technical capacity to interpret and use evidence.
We used data extracted from facility registers in 2425 facilities in Ethiopia to verify the accuracy of HMIS data for two services: visits for deliveries and caesarean sections (C-sections). We describe the magnitude of agreement between HMIS and source documents, and given our large facility sample, we also highlight some factors associated with good reporting. Finally, we describe our experience working with these data for research and policy translation. Our findings and recommendations may guide future data quality improvement efforts across LMICs.

\section{USING AN EXISTING SURVEY TO VERIFY THE ACCURACY OF HMIS DATA}

We used a survey conducted in 2016 by the Ethiopian Public Health Institute (EPHI) with support from the Averting Maternal Death and Disability Programme at Columbia University: the Ethiopian Emergency Obstetric and Newborn Care (EmONC) assessment. ${ }^{15}$

The 2016 EmONC assessment was a national crosssectional census of public and private health facilities that provided maternal and newborn health services in Ethiopia. It included information on infrastructure, human resources, facility service statistics and provider knowledge among others. The survey was conducted from April to December 2016. Data collectors identified labour and delivery ward and operating theatre paperbased registers and extracted the number of deliveries and C-sections performed for the 12 months of JanuaryDecember 2015. A labour and delivery register was available in all hospitals and maternal and child health $(\mathrm{MCH})$ specialty centres, and in $99 \%$ of health centres and clinics. The EmONC assessment included data from 3804 facilities in Ethiopia that provided maternal and newborn health services.

The numbers of deliveries and C-sections reported in the HMIS were compiled for the same months (JanuaryDecember 2015). At the time of the survey, Ethiopia used an electronic health management information systems (eHMIS) developed by John Snow. The DHIS2 was later adopted in 2018. Data from the eHMIS used in this study were accessed through the Ethiopian Health Data Analytics Platform. ${ }^{20}$ The number of births attended by health personnel was reported each month by all health facilities conducting deliveries in Ethiopia including health centres, primary hospitals, general hospitals, referral hospitals and private health facilities. C-sections were also reported monthly to the HMIS by all facilities with surgical capacity.

Because the two datasets did not contain common health facility identifiers or reliable geocodes (at the time of the survey), we created a statistical code to link health facilities in the EmONC assessment to the HMIS database using region, zone, woreda (ie, district), facility types and facility names. Merging was also checked manually and validated by local researchers. The dataset did not contain any patient-level or identifiable information. The 
Harvard Longwood Campus Institutional Review Board considers this study exempt from full review as it was based on anonymous, non-identifiable secondary data that did not involve human subjects.

Of the 3804 facilities included in the survey, 2479 $(65.2 \%)$ were successfully linked to the HMIS. However, labour and delivery registers were missing in 22 facilities and an additional 32 reported no deliveries for the year, leading to a sample of 2425 facilities. The majority of facilities were public health centres, and slightly more than half were in rural areas (table 1). The facilities had an average of 6.2 maternity beds and 26.5 total staff, and $72.3 \%$ were in Oromia or in the Southern Nations, Nationalities, and Peoples' Region (SNNP). Our analytical sample was similar to the full census in terms of facility characteristics but tended to include more facilities from certain regions (table 1). For example, Dire Dawa, Oromia and Addis Ababa regions were over-represented, while less than $40 \%$ of Beninshangul-Gumuz, Tigray, Amhara, Gambella and Afar facilities were included. Reasons for these regions being under-represented may include important differences in facility names between the HMIS and the survey or that more facilities did not report at all to the HMIS in these regions.

In 2015, these 2425 facilities reported a total of 1516 368 million deliveries to the HMIS, and 1423723 million deliveries were found in their registers (table 2). At the national level, this represented a $6.5 \%$ over-reporting in the HMIS. In contrast, 71542 C-sections were reported in the HMIS, but 74061 were found in operating theatre registers, an under-reporting of $-3.4 \%$ at the national level. Somali and Afar had the highest over-reporting, whereby $47.0 \%$ and $34.3 \%$ more deliveries were reported to the HMIS compared with those found in registers, respectively. Somali also over-reported C-sections by 27.3\%. Addis Ababa and Tigray under-reported both services (table 2).

\section{VERIFICATION FACTORS}

We created a VF at the facility level calculated as the number of deliveries (or C-sections) reported in the HMIS divided by the number of deliveries (or C-section) found in registers for the year. The resulting ratio was equal to 1 when totals in HMIS and registers were identical, greater than 1 when HMIS over-reported and below 1 when HMIS under-reported (The WHO's Data Quality Review toolkit calculates a VF by dividing the recounted number of events from source documents by the reported number from the HMIS (ie, registers/HMIS) ${ }^{13}$ For ease of interpretation, we opted to calculate the VF as HMIS divided by registers. With this method, VFs greater than 1 imply HMIS over-reporting, while VFs smaller than 1 imply under-reporting in the HMIS).

The VFs for deliveries (HMIS/registers) ranged from 0.05 (indicating that the number of deliveries in the HMIS was only $5 \%$ of that found in the registers) to 30.89 (indicating that the number of deliveries in the HMIS was 30.89 times greater than that found in registers), with a mean of 1.26
Table 1 Characteristics of facilities included in the study compared with the total facilities providing maternal and newborn health services in 2015 in Ethiopia

\begin{tabular}{|c|c|c|c|c|}
\hline \multirow[b]{2}{*}{ Facility types } & \multicolumn{2}{|c|}{ Study sample } & \multicolumn{2}{|c|}{ All facilities* } \\
\hline & $\mathbf{N}$ & $\%$ & $\mathbf{N}$ & $\%$ \\
\hline Hospitals $†$ & 232 & 9.6 & 293 & 7.7 \\
\hline Health centres & 2171 & 89.5 & 3459 & 90.9 \\
\hline $\begin{array}{l}\text { MCH specialised } \\
\text { centres }\end{array}$ & 22 & 0.9 & 52 & 1.37 \\
\hline \multicolumn{5}{|l|}{ Ownership } \\
\hline Public & 2350 & 96.9 & 3662 & 96.3 \\
\hline Private & 75 & 3.1 & 142 & 3.7 \\
\hline \multicolumn{5}{|l|}{ Location } \\
\hline Rural & 1385 & 57.1 & 2307 & 60.7 \\
\hline Urban & 1040 & 42.9 & 1497 & 39.4 \\
\hline $\begin{array}{l}\text { Infrastructure and } \\
\text { staffing }\end{array}$ & Median & IQR & Median & IQR \\
\hline $\begin{array}{l}\text { Number of } \\
\text { maternity beds }\end{array}$ & 4 & 3 & 4 & 3 \\
\hline Total staff $\ddagger$ & 14 & 10 & 14 & 9 \\
\hline Regions & $\mathrm{N}$ & $\%$ & $\mathrm{~N}$ & $\%$ \\
\hline Addis Ababa & 129 & 5.3 & 151 & 4.0 \\
\hline Afar & 30 & 1.2 & 77 & 2.0 \\
\hline Amhara & 295 & 12.2 & 876 & 23.0 \\
\hline Benishangul-Gumuz & 11 & 0.5 & 43 & 1.1 \\
\hline Dire Dawa & 19 & 0.8 & 21 & 0.6 \\
\hline Gambella & 10 & 0.4 & 27 & 0.7 \\
\hline Harari & 8 & 0.3 & 15 & 0.4 \\
\hline Oromia & 1267 & 52.3 & 1405 & 36.9 \\
\hline SNNPR & 486 & 20.0 & 773 & 20.3 \\
\hline Somali & 94 & 3.9 & 161 & 4.2 \\
\hline Tigray & 76 & 3.1 & 255 & 6.7 \\
\hline Total & 2425 & & 3804 & \\
\hline
\end{tabular}

${ }^{*}$ Census of all facilities that provided maternal and newborn health services in 2015 in Ethiopia. Surveyed by the 2016 Ethiopian Emergency Obstetric and Newborn Care assessment. †Hospital types include referral, general and primary hospitals. $\mathrm{MCH}$ specialised centres also include higher clinics. $\ddagger$ Total staff includes all health staff currently employed by the facility (medical doctors, obstetricians, gynecologists, pediatricians, neonatologists, emergency surgical officer, midwives, nurses, health officers, anesthesiologists, laboratory technicians, pharmacists and health information technologists). $\mathrm{MCH}$, maternal and child health; SNNPR, Southern Nations, Nationalities, and Peoples' Region.

and a median of 1.03 (figure 1). Across the 208 health facilities that performed C-sections, the VF ranged from 0.30 to 4.00 , with a mean of 0.98 and a median of 1.00 .

We also created two binary indicators based on the VF for good and very good reporting. Facilities were categorised as having 'good reporting' if the HMIS was within $10 \%$ of source documents (VF ranges from 0.90 
Table 2 Total deliveries and C-sections reported in the Ethiopian HMIS compared with those reported in facility registers in 2425 facilities, by region, in 2015

\begin{tabular}{|c|c|c|c|c|c|c|}
\hline & \multicolumn{3}{|l|}{ Deliveries } & \multicolumn{3}{|c|}{ C-sections } \\
\hline & HMIS & Registers & $\%$ difference & HMIS & Registers & $\%$ difference \\
\hline Addis Ababa & 89962 & 94562 & $-4.9 \%$ & 23133 & 25076 & $-7.8 \%$ \\
\hline Afar & 5098 & 3795 & $34.3 \%$ & 130 & 129 & $0.8 \%$ \\
\hline Amhara & 167610 & 151628 & $10.5 \%$ & 9754 & 10318 & $-5.5 \%$ \\
\hline Benishangul-Gumuz & 5842 & 5764 & $1.4 \%$ & 714 & 700 & $2.0 \%$ \\
\hline Dire Dawa & 8988 & 8184 & $9.8 \%$ & 1336 & 1299 & $2.9 \%$ \\
\hline Gambella & 2620 & 2435 & $7.6 \%$ & 120 & 135 & $-11.1 \%$ \\
\hline Harari & 5662 & 5301 & $6.8 \%$ & 930 & 908 & $2.4 \%$ \\
\hline Oromia & 841731 & 796264 & $5.7 \%$ & 19212 & 18917 & $1.6 \%$ \\
\hline SNNPR & 311603 & 284023 & $9.7 \%$ & 10976 & 10835 & $1.3 \%$ \\
\hline Somali & 23726 & 16138 & $47.0 \%$ & 621 & 488 & $27.3 \%$ \\
\hline Tigray & 53526 & 55629 & $-3.8 \%$ & 4616 & 5256 & $-12.2 \%$ \\
\hline National & 1516368 & 1423723 & $6.5 \%$ & 71542 & 74061 & $-3.4 \%$ \\
\hline
\end{tabular}

C-section, caesarean section; HMIS, health management information systems; SNNPR, Southern Nations, Nationalities, and Peoples' Region.

to 1.10). 'Very good reporting' included facilities that reported within $5 \%$ of source documents ( $\mathrm{VF}$ ranges from 0.95 to 1.05 , a narrower criterion). For deliveries, we found that two-thirds of facilities $(65.7 \%)$ had good HMIS reporting (VF ranges from 0.90 to 1.10) (figure 1). Among the remaining $34.3 \%$ of facilities, $5.0 \%$ underreported and $29.3 \%$ over-reported by more than $10 \%$.
Half of facilities $(50.5 \%)$ had very good HMIS reporting (VF ranges from 0.95 to 1.05 ). For C-sections, $60.1 \%$ of facilities had good HMIS reporting and $42.8 \%$ had very good reporting.

In figure 2, we summarised the VFs for deliveries by region. Median VFs were within 5\% of source documents (very good reporting) in six regions and greater than
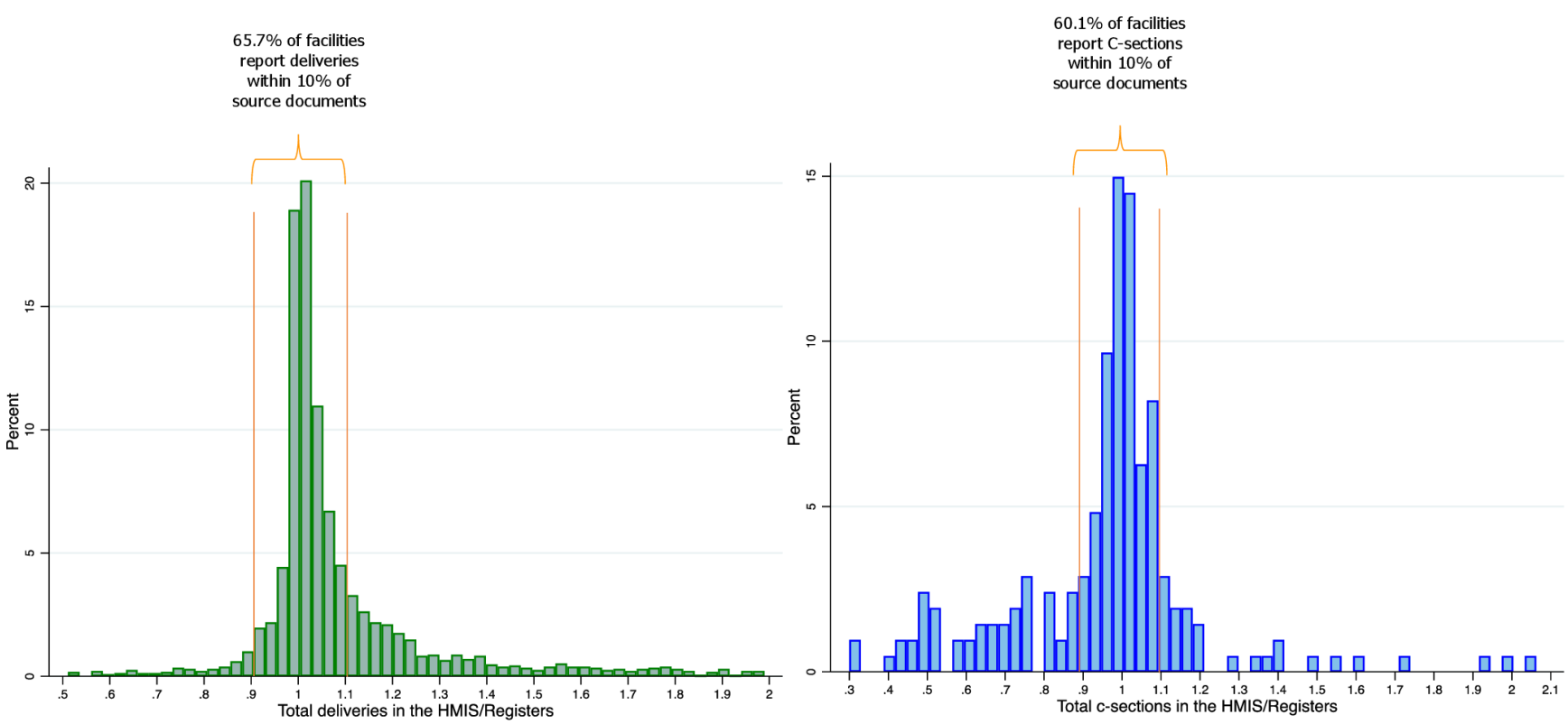

Figure 1 Ratios of deliveries and C-sections reported in the Ethiopian HMIS to those reported in paper-based facility registers (verification factors) by facilities in 2015. HMIS, health management information systems; C-section, Caesarean section. Ratios of the total deliveries and C-sections reported in the HMIS from January to December 2015 to that extracted from health facility registers for the same months (verification factors (VFs)). A VF of 1 means that the number of deliveries in the HMIS matches that found in registers. A VF $>1$ indicates a greater number reported in the HMIS, and a VF $<1$ indicates a smaller number reported in the HMIS compared to registers. Histogram on the left is truncated at 0.5 and 2.0 to show the region of the plot with most facilities (147 out of 2,425 facilities $(6 \%)$ are excluded). The plot on the right excludes one facility with a VF of 4 for C-sections. 


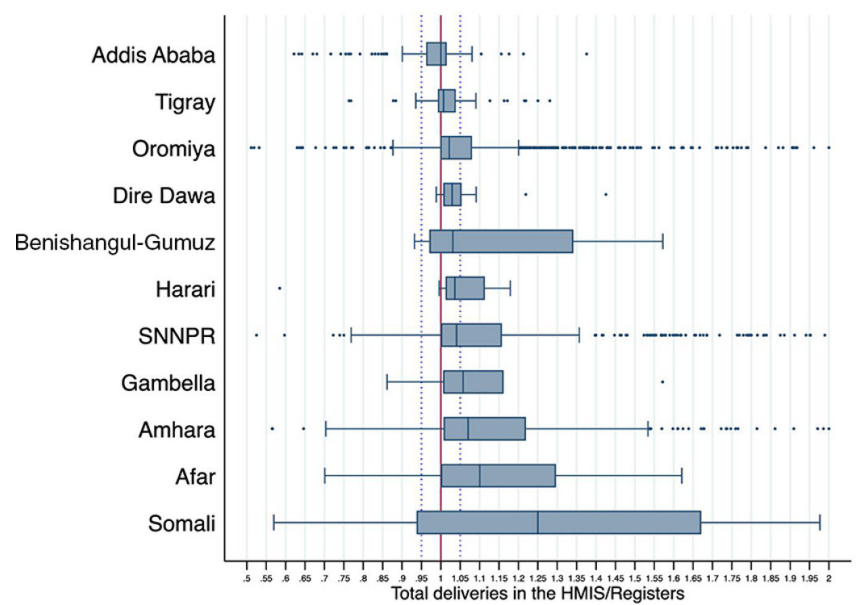

Figure 2 Verification factors (total deliveries in HMIS divided by that in registers) by Ethiopian region in 2015 . HMIS, health management information systems; SNNPR, Southern Nations, Nationalities, and Peoples' Region. Boxplots are truncated at 0.5 and 2.0 to show the region of the plot with most facilities (147 of 2,425 facilities (6\%) are excluded). Regions are sorted by median VF after truncation. The red line at 1 indicates perfect agreement. The blue dotted lines show the region of "very good" reporting: HMIS is within $5 \%$ of registers. The median VF in BenishangulGumuz was 1.17 before truncation.

1.05 in five regions. Somali and Afar, in particular, had the most over-reporting.

\section{FACILITY CHARACTERISTICS ASSOCIATED WITH GOOD REPORTING}

To explore potential factors associated with good reporting we used multivariable logistic regression models with robust standard errors, regressing the binary indicator for good reporting (reporting within $10 \%$ of source documents) on a series of facility characteristics: facility type (hospital, health centre and MCH specialised centre), ownership (public vs private), location (urban vs rural), and region fixed effects. We repeated the regression using the indicator for very good reporting (reporting within 5\% of source documents). We removed outliers by excluding the top and bottom $1 \%$ of facilities based on the VF estimates. ${ }^{21}$ The analysis thus included 2376 facilities with VFs ranging from 0.47 to 6.02.

We found that the odds of good reporting were higher in urban compared with rural areas (OR: 1.30, 95\% CI 1.06 to 1.59 ), in public compared with private facilities (OR: 2.95, 95\% CI 1.38 to 6.29 ) and in hospitals compared with health centres (OR: 1.71, 95\% CI 1.13 to 2.61) (table 3). Compared with Addis Ababa (the capital region), nearly all regions had lower odds of good and very good reporting.

\section{CHALLENGES AND RECOMMENDATIONS Data elements from HMIS should be used more broadly for policy and research}

Other studies have verified HMIS data elements in Ethiopia using source documents. In 2016, the EPHI and the Federal Ministry of Health (FMOH), with technical assistance from the WHO, conducted a data verification study using source documents from approximately 400 facilities, including all hospitals and a representative sample of health centres and private clinics. ${ }^{8}$ The verification covered seven HMIS data elements reported over 3 months (July-September 2015). The study found mean

Table 3 Odds ratios for the associations between facility characteristics and 'good' and 'very good' HMIS reporting (HMIS and paper-based registers are within $10 \%$ and $5 \%$, respectively)

\begin{tabular}{|c|c|c|c|c|c|c|c|c|c|}
\hline & \multirow[b]{2}{*}{$\mathbf{N}$} & \multicolumn{4}{|c|}{ Good reporting (VF ranges from 0.90 to 1.10 ) } & \multicolumn{4}{|c|}{ Very good reporting (VF ranges from 0.95 to 1.05} \\
\hline & & OR & $95 \% \mathrm{Cl}$ & & $P$ value & OR & $95 \% \mathrm{Cl}$ & & $P$ value \\
\hline \multicolumn{10}{|l|}{ Facility type* (ref. health centres) } \\
\hline $\mathrm{MCH}$ specialised centres & 21 & 0.80 & 0.25 & 2.57 & 0.707 & 0.58 & 0.18 & 1.84 & 0.358 \\
\hline Public ownership (ref. private) & 2307 & 2.95 & 1.38 & 6.29 & 0.005 & 2.64 & 1.30 & 5.38 & 0.007 \\
\hline Afar & 24 & 0.09 & 0.03 & 0.24 & 0.000 & 0.09 & 0.03 & 0.25 & 0.000 \\
\hline Amhara & 292 & 0.20 & 0.11 & 0.36 & 0.000 & 0.23 & 0.14 & 0.38 & 0.000 \\
\hline Benishangul-Gumuz & 11 & 0.15 & 0.04 & 0.51 & 0.002 & 0.18 & 0.05 & 0.66 & 0.010 \\
\hline Dire Dawa & 19 & 0.74 & 0.22 & 2.46 & 0.617 & 0.59 & 0.22 & 1.61 & 0.303 \\
\hline Somali & 77 & 0.03 & 0.01 & 0.08 & 0.000 & 0.01 & 0.00 & 0.05 & 0.000 \\
\hline Tigray & 76 & 1.06 & 0.46 & 2.43 & 0.884 & 1.07 & 0.53 & 2.17 & 0.842 \\
\hline
\end{tabular}

${ }^{*}$ Hospital types include referral, general and primary hospitals. $\mathrm{MCH}$ specialised centres also include higher clinics.

HMIS, health management information systems; $\mathrm{MCH}$, maternal and child health; ref., reference; SNNPR, Southern Nations, Nationalities, and Peoples' Region; VF, verification factor. 
VFs (defined as source documents divided by HMIS) of 0.92 for antenatal care visits, 1.01 for deliveries, 0.96 for the third dose of pentavalent vaccine, 0.95 for prevention of mother-to-child transmission of HIV, 0.95 for tuberculosis, 0.92 for malaria and 0.80 for family planning. This indicates that, for the exception of family planning, all indicators were reported within $10 \%$ of source documents on average. Endriyas and colleagues conducted a verification in 163 facilities in the SNNPR. ${ }^{6}$ They found that the proportion of facilities reporting accurately (within 10\% of source documents) ranged from $46.6 \%$ for the number of fourth antenatal care visits to $84.7 \%$ for deliveries and $96.9 \%$ for maternal deaths. ${ }^{6}$

Data elements in the HMIS include the absolute numbers of services delivered, of consultations taking place, and headcounts, cases and deaths from particular conditions. Although some work remains to perfect reporting, we believe that our findings and those of the studies cited previously support greater use and uptake of HMIS data elements for research and policy decisions in Ethiopia. ${ }^{6}{ }^{8}$ Furthermore, these studies were conducted using data from 2015 to 2017. HMIS quality has likely improved since, given the multitude of training initiatives and interventions to improve data quality that have recently taken place in Ethiopia. ${ }^{52-24}$ The expansion of internet connectivity along with the adoption of DHIS2 in 2018 may have facilitated standardisation in reporting and further improved data quality in Ethiopia.

\section{Data quality improvement should target the private sector, smaller facilities and those in rural areas}

Reasons for inconsistent HMIS reporting in LMICs remain unclear and likely differ across facility types, indicator types, regions and countries. In our study, private facilities had poorer reporting and should be prioritised for HMIS data quality interventions. Lack of any HMIS reporting or poor-quality reporting by the private sector has been found in other countries. ${ }^{18} 2526$ There is an urgent need to develop incentives for private facilities to report more accurately to national HMIS.

Hospitals also had better quality reporting than health centres. ${ }^{15}$ Overall, the number of C-sections tended to be better reported than routine deliveries, likely because they are conducted primarily in hospitals. Hospitals are generally better equipped and employ more staff for record keeping and data reporting. ${ }^{27}$ The EmONC assessment found that only $66 \%$ of health centres in Ethiopia had a dedicated person responsible for maternal and newborn health data reporting (such as a data manager or health information technologist) compared with 90\%-100\% in hospitals. ${ }^{15}$ As a subanalysis, we regressed the binary indicators for good and very good reporting on urban area, presence of a designated data manager and region fixed effects for health centres only $(\mathrm{N}=2103)$. We found that the odds of good and very good reporting were 1.55 and 1.24 times higher, respectively, in health centres with a dedicated data manager (data not shown). Ensuring that each facility has a dedicated staff for managing and reporting health service data would be a valuable strategy to improve HMIS accuracy.

Facilities in Somali and Afar had the lowest odds of good reporting and were substantially more likely to over-report in the HMIS (table 2 and figure 2). These two regions are predominantly rural and inhabited by pastoral communities. Though it is unclear why they tend to over-report, facilities in these rural areas face structural challenges that may affect HMIS reporting, including lack of reliable electricity or computers. Basic infrastructure is necessary for HMIS reporting in a digital format.

\section{Population coverage indicators from HMIS may not be reliable yet}

Other studies have compared population coverage indicators between the Ethiopian HMIS and DHS and found very low agreement. Adane and colleagues estimated that the proportion of fully vaccinated children in Ethiopia would be $89 \%$ in the HMIS but only $39 \%$ in the DHS. The Ethiopian FMOH also routinely compares health intervention coverage using the HMIS to those from the DHS. ${ }^{28}$ Coverage estimates from HMIS often differ from household survey estimates, and it is not uncommon to find coverage estimates that are greater than $100 \%$. Coverage indicators (reported as proportions) require both valid numerators (eg, number of fully vaccinated children aged 1 year) and denominators (total number of 1-year-old children). Discrepancies in coverage measures between HMIS and population-based surveys are the product of both inaccurate service volumes (numerators) and inaccurate target populations (denominators). Target populations are estimated using national census data on population age and sex, which are projected using assumptions about fertility, mortality and migration. In Ethiopia, the latest population census was conducted in $2007 .{ }^{29}$ A 2018 study across 14 Eastern and Southern African countries found that the median year of the most recent census used for population projections was 2009. ${ }^{11}$ The same study also found inaccuracies in population projection calculations and inappropriate use of population growth rates and crude birth rates. ${ }^{11}$ Without accurate population projections, the HMIS may not produce accurate coverage estimates.

\section{STRENGTHS AND LIMITATIONS OF THIS STUDY}

Our study is one of a few to verify the accuracy of HMIS data elements and provides a framework and methods for others to assess HMIS data quality. Unlike other data quality studies, we were able to include a large number of facilities $(\mathrm{N}=2425)$, which allowed us to investigate the facility characteristics associated with good reporting. Nonetheless, our study presents several limitations. First, we used data from paper-based registers as the gold standard to verify HMIS data. These source documents may also be incomplete or may contain errors. Nonetheless, our study simply relied on tallying the number of deliveries and C-sections performed and did not include 
detailed patient information from source documents. Second, our analysis includes only two data elements from the HMIS (deliveries and C-sections). The Ethiopian HMIS contains over 100 data elements and indicators on volumes of services (including reproductive, maternal, newborn and child health services, vaccinations, tuberculosis care, HIV care and chronic disease care). It also includes quality-of-care indicators (eg, the proportion of asphyxiated neonates who were resuscitated and survived, and the proportion of hypertensive patients with controlled blood pressure) and institutional deaths (eg, maternal, neonatal and inpatient deaths). The precision of other indicators may differ, especially those with more complex definitions or that are more complex to measure. Nonetheless, other studies have found similar reporting quality for other HMIS data elements in Ethiopia. ${ }^{68}$ Third, our study includes only $64 \%$ of all facilities that provided maternal and newborn care in Ethiopia at the time. The other $36 \%$ could not be linked between the two datasets, likely due to differences in facility or woreda names or because they did not report to the national HMIS. Though our sample was largely consistent with the facility census in terms of facility characteristics, certain regions were over-represented. For example, we were able to link $85 \%$ and $90 \%$ of all facilities in Addis Ababa and Dire Dawa, but only 58\% and 39\% of all facilities in Somali and Afar. Our results may therefore slightly overestimate data quality at the national level. Finally, the EmONC assessment did not include health posts. Health posts, staffed with community health workers, are the lowest levels of the primary healthcare system. Although health posts are not supposed to conduct deliveries, it is possible that some women gave birth at these facilities.

\section{CONCLUSIONS}

Across a large sample of health facilities in Ethiopia, we found that two-thirds of facilities had good HMIS reporting for institutional deliveries (defined as reporting within $10 \%$ of source documents for the year). Urban facilities, hospitals and public facilities had higher odds of good reporting. The Somali and Afar regions had the most over-reporting. Despite the limitations stated earlier, our results and those of similar verification studies support greater use and uptake of HMIS data for research and policy decisions in Ethiopia. Increased use of these data, combined with feedback mechanisms, is necessary to improve and maintain data quality. Rather than relying heavily on externally driven surveys conducted once every 5 years, Ethiopia and countries with similar HMIS must invest in building local capacity for reporting and analysing HMIS data. The adoption of the DHIS2, a web-based standardised HMIS platform, across 73 LMICs offers a renewed opportunity for global efforts to contribute to improving these data systems. ${ }^{101130}$

Future research should further explore and address the underlying causes of poor HMIS reporting in certain areas. Efforts to improve HMIS should also consider reviewing the total number of data elements required for reporting by facilities every month, the clarity of reporting guidance and definitions, and the estimation of target population size used by the HMIS to estimate coverage indicators. Moving forward, we conclude that country-led HMIS should be the primary source of information for tracking health system performance in LMICs.

Contributors CA and SV designed the study. CA and BY linked the datasets. CA performed the analyses and wrote the first draft of the paper. GD and MK contributed to the paper design, interpretation of results and recommendations. All authors read, improved and approved the final manuscript.

Funding We thank the Ethiopian Public Health Institute for providing the 2016 EmONC assessment dataset and the Ministry of Health for providing access to the Ethiopian Health Data Analytics Platform. We also thank Frehiwot Getachew and Min Kyung Kim for their support in linking datasets. We acknowledge funding from the Bill \& Melinda Gates Foundation through the Fenot project (INV-010189) and the Disease Control Priorities-Ethiopia project (INV-010174).

Competing interests None declared.

Patient consent for publication Not required.

Provenance and peer review Not commissioned; externally peer reviewed.

Data availability statement Data may be obtained from a third party and are not publicly available.

Open access This is an open access article distributed in accordance with the Creative Commons Attribution 4.0 Unported (CC BY 4.0) license, which permits others to copy, redistribute, remix, transform and build upon this work for any purpose, provided the original work is properly cited, a link to the licence is given, and indication of whether changes were made. See: https://creativecommons.org/ licenses/by/4.0/.

\section{ORCID iDs}

Catherine Arsenault http://orcid.org/0000-0003-2410-3015

Stéphane Verguet http://orcid.org/0000-0003-4128-0849

\section{REFERENCES}

1 Farnham A, Utzinger J, Kulinkina AV, et al. Using district health information to monitor sustainable development. Bull World Health Organ 2020;98:69-71.

2 Hung YW, Hoxha K, Irwin BR, et al. Using routine health information data for research in low- and middle-income countries: a systematic review. BMC Health Serv Res 2020;20:790.

3 The Federal Democratic Republic of Ethiopia Ministry of Health. Health sector transformation plan 2015/16-2019/20 (2008-2012 EFY), Addis Ababa, Ethiopia 2015.

4 Mulissa Z, Wendrad N, Bitewulign B, et al. Effect of data quality improvement intervention on health management information system data accuracy: an interrupted time series analysis. PLoS One 2020;15:e0237703.

5 Adane A, Adege TM, Ahmed MM, et al. Routine health management information system data in Ethiopia: consistency, trends, and challenges. Glob Health Action 2021;14:1868961.

6 Endriyas M, Alano A, Mekonnen E, et al. Understanding performance data: health management information system data accuracy in Southern Nations Nationalities and People's Region, Ethiopia. BMC Health Serv Res 2019;19:175.

7 Ouedraogo M, Kurji J, Abebe L, et al. A quality assessment of health management information system (HMIS) data for maternal and child health in Jimma zone, Ethiopia. PLoS One 2019;14:e0213600.

8 Ethiopian Public Health Institute, Federal Ministry of Health of Ethiopia, The World Health Organization. Ethiopia health data quality review: system assessment and data verification 20162016.

9 Bhattacharya AA, Umar N, Audu A, et al. Quality of routine facility data for monitoring priority maternal and newborn indicators in DHIS2: a case study from Gombe state, Nigeria. PLoS One 2019;14:e0211265.

10 Nshimyiryo A, Kirk CM, Sauer SM, et al. Health management information system (HMIS) data verification: a case study in four districts in Rwanda. PLoS One 2020;15:e0235823.

11 Maïga A, Jiwani SS, Mutua MK, et al. Generating statistics from health facility data: the state of routine health information systems in eastern and southern Africa. BMJ Glob Health 2019;4:e001849. 
12 O'Hagan R, Marx MA, Finnegan KE, et al. National assessment of data quality and associated Systems-Level factors in Malawi. Glob Health Sci Pract 2017;5:367-81.

13 World Health Organization. Data quality review: a toolkit for facility data quality assessment. module 2 . Desk review of data quality 2017.

14 World Health Organization. Analysing and using routine data to monitor the effects of COVID-19 on essential health services: practical guide for national and subnational decision-makers 2021.

15 Ethiopian Public Health Institute, Federal Ministry of Health, Averting Maternal Death and Disability, Columbia University. Ethiopian emergency obstetric and newborn care assessment 2016, Addis Ababa, Ethiopia 2017.

16 USAID, MEASURE EValuation, SNNP Regional Health Bureau. Health Management Information System (HMIS) Facilitator's Guide for Training of Trainers. Version 1.12010.

17 Kebede M, Adeba E, Chego M. Evaluation of quality and use of health management information system in primary health care units of East Wollega zone, Oromia regional state, Ethiopia. BMC Med Inform Decis Mak 2020;20:107.

18 Rumisha SF, Lyimo EP, Mremi IR, et al. Data quality of the routine health management information system at the primary healthcare facility and district levels in Tanzania. BMC Med Inform Decis Mak 2020;20:340.

19 Harvard TH. Chan School of Public Health and the School of Population and Public Health, University of Bristish Columbia. strengthening primary health care systems performance in lower and middle-income countries, the Fenot project: Fenot: achieving excellence in primary care, 2021. Available: https://fenot.bcchdigital. $\mathrm{ca} /$
20 Federal Democratic Republic of Ethiopia. Health sector transformation PLAN-I, annual performance report, EFY 2009 (2016/17) 2017.

21 Iglewicz B, Hoaglin DC. How to detect and handle outliers. Mexico: Asq Press, 1993.

22 Argaw MD, Desta BF, Kibret MA, et al. Accelerating the performance of district health systems towards achieving UHC via twinning partnerships. BMC Health Serv Res 2020;20:892.

23 Bogale A. Implementation status of health management information system in hospitals of South West Shoa zone, Oromia, central Ethiopia. Clinicoecon Outcomes Res 2021;13:1-8.

24 Endriyas M, Alano A, Mekonnen E, et al. Decentralizing evidencebased decision-making in resource limited setting: a case of SNNP region, Ethiopia. PLoS One 2020;15:e0236637.

25 MEASURE Evaluation. Health information systems strengthening Resouce center, Country Profiles, 2017. Available: https://www. measureevaluation.org/his-strengthening-resource-center/countryprofiles-1.html

26 Begum T, Khan SM, Adamou B, et al. Perceptions and experiences with district health information system software to collect and utilize health data in Bangladesh: a qualitative exploratory study. BMC Health Serv Res 2020;20:465.

27 Ethiopian Public Health Institute, Federal Ministry of Health. Ethiopia service availability and readiness assessment (SARA) Addis Ababa, Ethiopia 2018.

28 Federal Democratic Republic of Ethiopia, Ministry of Health, Policy Planning Directorate. Health and health related indicators, EFY 2010 (2017/18) 2018.

29 Central Statistical Authority of Ethiopia. 2007 population and housing census of Ethiopia, administrative report. Addis Ababa 2012.

30 dhis2. About DHIS2, 2021. Available: https://www.dhis2.org/about 